\title{
Urgences
}

\section{La variante en regard de la structure générale du texte : sur deux nouvelles d'André Major}

\section{Noël Audet et André Carpentier}

Numéro 24, juillet 1989

Le manuscrit sous l'angle

URI : https://id.erudit.org/iderudit/025533ar

DOI : https://doi.org/10.7202/025533ar

Aller au sommaire du numéro

Éditeur(s)

Urgences

ISSN

0226-9554 (imprimé)

1927-3924 (numérique)

Découvrir la revue

Citer cet article

Audet, N. \& Carpentier, A. (1989). La variante en regard de la structure générale du texte : sur deux nouvelles d'André Major. Urgences, (24), 67-75.

https://doi.org/10.7202/025533ar d'utilisation que vous pouvez consulter en ligne.

https://apropos.erudit.org/fr/usagers/politique-dutilisation/ 


\section{La variante en regard de la structure générale du texte: sur deux nouvelles d'André Major}

Noël Audet et André Carpentier, Université du Québec à Montréal

On ne peut embrasser les ouvrages de la nature et de l'art quand ils sont achevés; il faut les saisir au vol, à l'état naissant, si l'on veut parvenir à les comprendre.

Johann Wolfgang von Gœthe

La critique génétique, qui vise à reconstituer la chaîne avanttextuelle, offre le tableau d'une mise en place hypothétique, parfois probable, du texte; elle propose un récit du texte, en donnant à lire un itinéraire de sa composition. La manipulation dévoilée des signes de l'avant-texte permet en effet d'approcher le procès d'encodage, dans une mesure immensurable, et met en lumière, c'est ce à quoi nous nous intéresserons ici, la mouvance de l'intentionnalité du scripteur. Cette intentionnalité ne progresse pas, dirions-nous, mais bouge plutôt, jusqu'à se fixer dans la reconnaissance d'une possible finitude ${ }^{1}$ parmi d'autres qui la traversent.

Nous nous proposons, ici, d'illustrer cette mouvance en montrant que la microstructure et la structure globale d'un texte, contre l'impression généralement laissée par l'observation de ses états définitifs seuls, loin de révéler la fixité de procédés arrêtés, offrent, dans les avant-textes, le spectacle de leur

1 Rappelons les distinctions apportées par Raymonde DebrayGenette entre finitude: «le texte, comme dernier état arrêté, que ce soit par la volonté de l'écrivain ou par le hasard n; finition: «le texte comme point de perfection, ou même simplement de saturation »; et finalité: ale texte comme lieu d'accomplissement de projets définis à l'avance, mentalement ou ouvertement». Essais de critique génétique, "Génétique et poétique: le cas Flaubert", coll. "Textes et manuscrits", Paris, Flammarion, série publiée par Louis Hay, 1979, p. 25. 
développement; aussi, nous examinerons la figure d'interaction de ces micro et macrostructures.

Nous allons en la circonstance centrer notre curiosité sur des cas de variances ${ }^{2}$ dans deux nouvelles tirées du recueil $L a$ folle d'Elvis" d'André Major: "Ceux qui attendent" et "L'égarement". Nous comptons, pour mener cette observation, sur des avant-textes qui ne paraissent pas trop lacunaires: des brouillons (des états provisoires successifs) et le manuscrit ('état définitif) de La folle d'Elvis, et plus précisément, trois états de "Ceux qui attendent" et quatre de "L'égarement".

\section{«Ceux qui attendent ${ }^{4}$}

Voici l'histoire abrégée de "Ceux qui attendent". Dans un snack-bar près d'une gare, une serveuse sert des cafés à un homme qui attend l'heure de son train de retour vers Ottawa. La nouvelle est divisée en deux parties. La première est focalisée sur la serveuse; la seconde, sur le client. Les deux personnages se désirent, mais ne s'avouent pas leur sentiment. On reconnaît là les thèmes de l'incommunicabilité et du désir insatisfait omniprésents dans l'œuvre de Major.

Les brouillons de "Ceux qui attendent" révèlent à l'évidence les indices d'opérations de relecture et de quelques modifications en découlant qui ont permis à Major de consolider l'économie générale de son texte et qui lui ont fourni les paramètres indispensables pour procéder à des ajustements ou remaniements du texte par rapport à cette nouvelle donne.

Déjà le titre, "Ceux qui attendent", figurant en tête du premier état, impose une contrainte formelle: le récit doit utiliser les temps de l'attente, ceux qui marquent le mieux la durée intérieure grâce à l'aspect d'inaccompli qui est inscrit dans leur structure même, soit le présent, l'imparfait et le conditionnel. De même, comme it

2 Le mot est pris au sens de modifications qui interviennent entre des états manus/tapuscrits. Cette variation des signes donne à voir le passage de la pensée au langage.

3 André Major, La folle d'Elvis, nouvelles, Montréal, Québec/ Amérique, 1981, $137 \mathrm{p}$.

4 Le premier état de "Ceux qui attendent o date de mars 1979, le deuxième de l'automne de 1980. (Environ dix-huit mois les séparent donc.) Le deuxième état a servi à établir la version finale sous forme de dactylogramme - mis au net par Ginette Major. 
s'agit d'exploiter, sur le plan thématique, le désir dans sa double phase désirante et déceptive, et la tension qui en résulte, Major opte pour le monologue intérieur, dont le temps principal est aussi le présent et où le conditionnel et le futur servent à traduire l'espace du rêve. Le narrateur s'installe d'abord dans la conscience de celle qui attend que l'autre fasse les premiers pas, puis dans la conscience de celui qui attend parallèlement un semblable geste, les deux personnages rêvant d'une possible réalisation.

Or en lisant les brouillons, on constate que d'un état à l'autre les temps des verbes ont bougé. Dans l'état I, il y a déjà hésitation entre le passé et le présent — on assiste à quelques remplacements en surcharge; dans l'état $I I$, les choses se présentent plus clairement. 5

État I (folios 3-4):

[...] et puis elle en avait jusque-là de savoir que les autres finissaient toujours par rencontrer l'homme de leur vie, mais pas elle. Des ivrognes ou des malades qui voulaient passer la nuit dans son lit, ça, elle ne les /comptait $\rightarrow$ compte/ plus. Les hommes qui valaient la peine qu'on couche avec, ils lavaient $\rightarrow$ ont/ autre chose à faire [...]

$(\rightarrow)$ État II (folio 2):

[...] et puis elle en a /assez $\rightarrow$ jusque-la/ de voir que les autres finissent toujours par rencontrer l'homme de leur vie, mais /jamais elle $\rightarrow$ elle jamais/. Des ivrognes ou des obsédés qui veulent passer la nuit dans son lit, elle ne les compte plus. Les hommes qui vaudraient la peine qu'on couche avec, ils ont autre chose à faire [...]

On voit que Major a mis à l'épreuve le passé et le présent, avant de saisir l'avantage qu'il pouvait tirer de ce dernier. On note aussi cette substitution de "vaudraient" à "valaient", par

5 Le protocole de transcription utilisé dans cet article est le suivant: le signe [...] circonscrit des mots biffés - sans rajouts; le signe <...> circonscrit des mots ajoutés - hors du principe de rature; (le double signe $\langle[.]$.$\rangle signifiera donc qu'un segment a été biffé puis remis, et$ $[<\ldots>]$ qu'un segment a été ajouté puis biffé, ainsi de suite;) le signe/.../ circonscrit les mots impliqués dans la rature; le signe $\rightarrow$ indique que le segment précédant cette flèche a été modifié en ce qui la suit, le signe $\Rightarrow$ désigne une deuxième modification, ainsi de suite; les mêmes signes entre parenthèses, $(\rightarrow),(=>)$, indiquent le passage du premier état du texte au deuxième, du deuxième au troisième, etc. 
laquelle l'héroïne se voit installée dans l'espace du désir; on lui fait tourner le dos à l'aspect d'accompli que charriait le passé. Dorénavant, elle sera attente pure, comme le titre le laisse présager. Par ces seuls changements de temps, la composition du texte vient de basculer vers ce qu'il devra devenir, compte tenu de la thématique de la déception qui se développe dans un double mouvement: construction du désir, puis sa constitution en objet de déception.

Pour que cette thématique s'accomplisse, il faut que le désir rencontre un objet désirable. Or, les séquences suivantes, dans les divers états, nous montrent sur cette question le remuement de la conscience structurante du scripteur, depuis une appréhension vague du devenir du texte jusqu'à une intention ferme. Le portrait bougé de cette structuration est perceptible dans les ratures, biffures et surcharges. ${ }^{6}$ L'objet du désir de l'héroïne est d'abord ainsi décrit:

\section{État I (folio 2):}

Lui, il n'était pas si mal, et même plutôt bien, seulement trop timide, trop peu sûr de lui pour qu'elle se sente portée vers lui. Elle n'aimait pas les fanfarons non plus.

Cet aveu, qui atténue le désir, a dû apparaître, à la relecture du premier état, comme une erreur compositionnelle, car s'il n'y a pas désir fort et réciproque, le reste du récit perd tout son sens. Cet aveu est donc contraire à l'économie générale du texte dans l'état de sa forme. L'intention du scripteur sera alors de faire en sorte que la déception n'intervienne plus dans l'ordre du désir, mais dans les événements seulement. À cette étape, donc, la conscience d'un certain aspect de la structure amène le scripteur à biffer, dans l'état II, les membres de phrase concernant précisément l'aveu de non-attirance: Major met sous biffure les deux unités de contenu suivants: "pour qu'elle se sente vraiment attirée par lui» et "Elle n'aimait pas les fanfarons non plus." La nouvelle version renverse la situation et produit de façon nette l'objet du désir. La timidité du voyageur, qui était d'abord un empêchement, devient pratiquement une qualité, surtout quand elle est comparée à l'attitude des fanfarons.

6 Biffure: «trait qui supprime un segment de l'écrit» (Jean BelleminNoël, Le texte et l'avant-texte, Paris, Librairie Larousse, 1972, p. 13). Rature: "Biffure à laquelle succède, en surcharge ou à la suite, une nouvelle formulation.» (ibid., p. 14). 


\section{État II (folio 2):}

Lui, il ne serait pas si mal, et même plutôt bien, seulement trop timide, trop peu sûr de lui. Les fanfarons courent les rues, mais elle ne peut pas les sentir.

Ces exemples tirés de l'avant-texte de "Ceux qui attendent" dévoilent en partie de quelle manière naît l'intentionnalité thématique chez Major. Ils montrent aussi que certaines variations de microstructure entraînent une modification de la macrostructure, qui à son tour, devenue autre et ne fonctionnant plus de la même manière, agit différemment sur ses parties. Cet effet réactionnel engendré dans l'organisme par son fonctionnement est donc de l'ordre de la rétroaction.

\section{«L'égarement»7}

"L'égarement" présente une histoire à deux volets. Le récit s'ouvre par une description de scène d'hiver. Antoine, un journaliste de province, fréquente une serveuse de restaurant, Marie-Rose, mais ne répond pas à l'intérêt amoureux qu'elle lui porte. Ici encore, comme dans "Ceux qui attendent", un accablement innommé empêche le personnage d'exprimer ses désirs et de vivre en concordance avec le monde. Parallèlement, un incendiaire sévit dans le village, et une nuit, tandis qu'Antoine est chez Marie-Rose, sa maison brûle. Antoine connaît le coupable depuis le début: il s'agit d'un menuisier à la retraite, autrefois unique ami de son grand-père. Un matin, on trouve le menuisier retraité mort de froid derrière la maison du boucher. La vie continue sous le signe de la banalité.

L'histoire de la composition de "L'égarement" paraît plus complexe que celle de "Ceux qui attendent", sans doute parce que cette nouvelle a connu, dès après le premier état, des modifications majeures sur les plans de la diégèse, du temps du récit, de la fonction des personnages, de la focalisation, etc. L'examen synchronique des brouillons permet, par ailleurs, d'apercevoir les signes d'un débat particulier qui accompagne et nourrit l'élaboration de "L'égarement", débat qui a des conséquences sur la composition.

7 Le premier état de "L'égarement date de janvier 1977; le deuxième, du 26 mai 1978; le troisième, de janvier 1980. (Les trois campagnes d'écriture de cette nouvelle s'étalent donc sur une période de trois ans.) Le troisième état a servi à la frappe définitive - qui est toujours le fait de Ginette Major. 
À la fin des années soixante-dix, en effet, selon son propre aveu maintes fois répété, André Major est en lutte pour éliminer la métaphore de son écriture. II dit lui-même, métaphoriquement! "pour assassiner la métaphore". Ce conflit au niveau des formes verbales, qui vise à qualifier le langage par une prise de position esthétique, se lit aisément dans des exemples contradictoires. Voici d'abord un cas de dé-métaphorisation portant sur une unité textuelle tirée d'une scène en forêt. ${ }^{8}$ Antoine marche sur une croûte de neige:

État I (folio 3):

Tout est pris dur, comme on dit ici. Dans le sentier [,] protégé des vents par [une] de grands pins, la neige résiste moins, on $<\left[s^{\prime}\right]>y$ enfonce de plusieurs pouces. Comme /e sentier $\rightarrow$ il/ serpente, je ne risque pas d'être découvert. Aucune odeur. Les troncs presque noirs dressés comme les barreaux d'une grandiose cellule jonchée de dunes d'une blancheur hallucinante.

$(\longrightarrow)$ État II (folio 3):

Tout est pris dur, comme on dit ici. Il s'agrippe aux broussailles et le voici sous les pins qui tendent leurs <larges> bras [énormes]. S'il se retournait, il [verrait qu'il] [les champs] serait ébloui par la blancheur des dunes.

(=>) État III (folio 2):

Tout est pris dur, comme on dit ici. S'il se retournait, il serait ébloui par la blancheur des dunes.

Ailleurs, et toujours sur la question de la blancheur, qui a valeur symbolique ${ }^{9}$ dans "L'égarement", on notera ce cas de métaphorisation:

État II (folio 2) ( $\longrightarrow$ ) état III (folio 2):

Il a bu du café devant la fenêtre qui donne sur les champs, attendant la /lumière $\longrightarrow$ lueur/ [toujours ${ }^{10}$ ] /blanche $\longrightarrow$ laiteuse/ de l'aube.

8 On remarquera également le travail de resserrement de l'écriture d'un état à l'autre.

9 Ce thème-symbole de la blancheur aboutira à la suppression de condensations sémantiques sexualisées dans une scène avec Pauline, la fille de Marie-Rose.

10 Biffé dès l'état II. Cette suppression participe d'une autre hésitation entre ponctuel et duratif. 
Ce que nous voudrions mettre en relief, dans ces exemples, c'est moins le travail autour de la métaphore que les répercussions de ce débat dans l'ordre du descriptif.

Dans le premier état, la narration est prise en charge par Antoine, qui est donc sujet de la focalisation; et l'incendiaire en est l'objet. Dès l'état II, la narration est assurée par un narrateur omniscient et Antoine devient l'objet de la focalisation. ${ }^{11}$ Or, le passage, entre l'état I et les suivants, du «je» narrateur-héros au “il» à vision extérieure neutre s'accompagne d'un travail sur la représentation qui continue de se développer dans les états II et III. Ce qui est mis en jeu, ici, c'est l'affaiblissement de la cause (par exemple: "la lumière") au profit de l'effet ("la lueur»), du descriptif ("il s'agrippe aux broussailles", "la lumière blanche") en faveur du perceptif ("la lueur laiteuse") ou de l'interprétatif ( "il serait ébloui"). C'est ainsi que paraît s'amoindrir, dans "L'égarement", sans s'éliminer tout à fait, la prédominance de la représentation sur la relation — du showing sur le telling, dans le langage de Percy Lubock.

On voit se former, dans ces exemples, des modèles transformationnels appliqués à des microsystèmes autres que thématiques; ce sont, cette fois, les systèmes rhétorique et descriptif qui sont modifiés, et surtout qui interagissent. On remarquera d'ailleurs que ces interactions de microstructures modifient également la composition générale dans sa forme du moment - comme dans l'exemple de "Ceux qui attendent"; puis que la macrostructure développe son potentiel de réverbération sur l'ensemble des microstructures. La transformation appelée par ces relations entre micro et macrostructure, quoique plus complexe que dans l'exemple tiré de "Ceux qui attendent", répond toujours à la figure de la rétroaction.

Les avant-textes de "Ceux qui attendent" et de "L'égarement" illustrent donc les rapports dialectiques des microstructures entre elles et de ces microstructures avec la

11 Les agendas d'André Major indiquent qu'à l'état I, la nouvelle s'intitule a L'incendiaire». Ce titre est conservé en tête de l'état II, mais dès lors reconnu inopérant, en raison du changement d'objet de focalisation, il est abandonné au profit de aL'égarement» lorsque s'ajoute en épigraphe ce mot de Cesare Pavese: aLe grand soleil répandait comme un égarement. (aLa prison», Avant que le coq chante [1949], «Folio», no 464, Paris, Gallimard, p. 122.) 
structure générale du texte, de même qu'entre ces objets se constituant dans et par le texte et l'intention de dire. Des structures (thématiques, narratives, esthétiques, etc.) se développent dans les avant-textes, se réorientent, se raffermissent et imposent à l'organisation du texte de nouvelles lois. Dans cette veine, on pourrait dire qu'un texte s'arrête en équilibre lorsque cesse ce mouvement qui vise à établir une étroite congruence entre l'expression et l'intentionnalité, entre le mot à mot du syntagme et la structure générale.

\section{La rétroaction}

La saisie d'une séquence dans les avant-textes permet d'approcher, en amont, "l'ouvrage en train de frayer sa voie à travers l'écrivain", selon le mot de Bellemin-Noël12, mais aussi, en aval, d'ajouter à l'interprétation du texte un témoignage sur son processus de production. Notons aussi que c'est dans le travail de l'écriture même, sur le contenu ou sur l'expression, par suppressions ou ajouts d'unités de désignation, par déplacements, etc., que s'élaborent les choix de désignation qui, à force, déterminent le champ de contenu. Et par rétroaction, les unités de contenu déterminent le champ de l'expression. C'est là à peu près ce que Mallarmé, il y a plus d'un siècle, appelait la "retrempe alternée en le sens et la sonorité". 13

Le critique qui veut faire le récit de cette retrempe, qui espère retracer les étapes et lieux scriptiques où le puzzle structural a bougé, où le texte s'est mis en place, qui souhaite retrouver l'oscillation de l'intentionnalité du scripteur, se donne le privilège de regarder dans la même direction que l'écrivain, pour voir aller le texte - plutôt que de le voir venir tout achevé. La tâche est fascinante mais difficile, car, comme le note Henri Mitterand:

[i]l ne s'agit pas d'aller d'un détail antérieur à un détail ultérieur, mais, autant que possible, d'une forme globale à

12 Jean Bellemin-Noël, Littérature, no 52, p. 125.

13 Stéphane Mallarmé, «Avant-dire au Traité du verbe» [1886], CEuvres complètes, texte établi et annoté par Henri Mondor et $G$. JeanAubry, coll. aBibliothèque de la Pléiade *, Paris, Gallimard, NRF, 1945, p. 361. Dix ans plus tard, Mallarmé reprend le mot, qui parle alors de «[...] la retrempe, d'ordinaire cachée, [qui] s'exerce publiquement, par le recours à de délicieux à-peu-près.» ( $\propto$ Crise de vers» [1896], Op. cit., p. 858.) 
celle qui lui succède, et, chaque fois, d'établir le modèle transformationnel global, aussi bien que les modèles des transformations secondaires dans l'ordre des contenus comme dans l'ordie des formes. ${ }^{14}$

Cela exige que les systèmes structuraux soient saisis conjointement, car c'est dans les effets réactionnels engendrés dans les unités de structure - c'est ce que veut dire rétroaction - par le fonctionnement rhizomatique de l'écriture qu'on voit le mieux bouger le texte, et se fixer.

14 Henri Mitterand, Essais de critique génétique, «Programme et préconstruit génétiques: le dossier de L'assommoir», coll. «Textes et manuscrits», Paris, Flammarion, série publiée par Louis Hay, 1979, p. 226. 